\title{
Flavonóides de Piper carniconnectivum C.DC.. Piperaceae
}

\author{
Harley da S. Alves, ${ }^{1}$ George E. de Oliveira, ${ }^{1}$ Maria das G. Zoghbi, ${ }^{2}$ Maria Célia de O. Chaves ${ }^{* 1}$
}

\author{
${ }^{1}$ Laboratório de Tecnologia Farmacêutica, Universidade Federal da Paraíba, Caixa Postal 5009, 58051-970 \\ João Pessoa-PB, Brazil \\ ${ }^{2}$ Museu Emílio Goeldi, 66077-830 Belém-PA, Brazil.
}

\begin{abstract}
RESUMO: Os flavonóides 5,3',4'-triidroxi-7-metoxiflavona, 5,3'-diidroxi-7-4'-dimetoxiflavona, 5,4'-diidroxi-7-metoxiflavona e uma mistura de esteróides ( $\beta$-sitosterol e estigmasterol) foram isoladas das partes aéreas de Piper carniconnectivum C.DC, Piperaceae. As estruturas desses compostos foram estabelecidas por análises espectroscópicas de UV, IV e RMN de ${ }^{1} \mathrm{H}$ e ${ }^{13} \mathrm{C}$, incluindo técnicas uni e bidimensionais.
\end{abstract}

Unitermos: Piper carniconnectivum, 5,3',4'-triidroxi-7-metoxiflavona, 5,3'-diidroxi-7-4'dimetoxiflavona, 5,4'-diidroxi-7-metoxiflavona, $\beta$-sitosterol, estigmasterol

\begin{abstract}
Flavonoids from Piper carniconnectivum C. DC. (Piperaceae)". The flavonoids 5,3',4'-trihydroxy-7-methoxyflavone, 5,3'-dihydroxy-7-4'-dimethoxyflavone, 5,4'-dihydroxy-7methoxyflavone and steroids mixture ( $\beta$-sitosterol and stigmasterol) were isolated from the dried aerials parts of Piper carniconnectivum C.DC. Their structures were established by spectroscopic analyses (UV, IV and ${ }^{1} \mathrm{H},{ }^{13} \mathrm{C}$ NMR, including 1D and 2D NMR).
\end{abstract}

Keywords: Piper carniconnectivum, 5,3',4'-trihydroxy-7-methoxyflavone, 5,3'-dihydroxy-7-4'dimethoxyflavone, 5,4'-dihydroxy-7-methoxyflavone, $\beta$-sitosterol, stigmasterol.

\section{INTRODUÇÃO}

Nas análises fitoquímicas de espécies de Piperáceas do Norte e Nordeste Brasileiro foram isoladas amidas (Araújo-Júnior et al, 1997; Santos et al, 1999a; Cunha et al, 2001; Chaves et al, 2003a; Chaves et al, 2003b), aristolactamas (Araújo-Júnior et al, 1999; Cardoso-Júnior et al, 2003 Chaves et al, 2006), fenilpropanóides (Santos et al, 1997; Santos et al, 1998a; Santos et al, 1999b, Chaves et al, 2002), fenilalcanóides (Santos et al, 1998b), terpenos e um derivado do ácido benzóico (Chaves et al, 2002).

Piper carniconnectivum C. DC., Piperaceae, conhecida popularmente como pimenta-longa, é uma espécie nativa da região Amazônica, Norte do Brasil (Yuncker, 1972). Um flavonóide (galangina), um fenilpropanóide (2-metoxi-4,5-metilenodioxipropiofenona), uma cumarina conhecida por xantiletina, três ciclopentenodionas e quatro flavonóides: 5-hidroxi-7-metoxi-6-metilflavanona, 5-hidroxi-7-metoxi-8-metilflavanona,5-hidroxi-7-metoxi6,8-dimetilflavanona e 2'-hidroxi-4',6'-dimetoxi-3',5'dimetilchalcona foram isolados nessa planta (Facundo et al, 2003; Facundo \& Braz-Filho, 2004).

Investigações fitoquímicas de espécies de Piperaceae têm mostrado variações distintas de flavonóides, especialmente aqueles com anel B não-substituído. Alguns flavonóides tri ou tetraoxigenados têm sido isolados (Parmar et al, 1997). Esse é o primeiro relato da ocorrência de 5,3',4'-triidroxi-7-metoxiflavona (1) e 5,4'-diidroxi-7metoxiflavona (3) em Piper. Esses compostos já foram isolados em várias outras famílias de plantas, tais como: (1) isolado em Asteraceae (Bohm, 1999), Lamiaceae (Ahmad et al, 2000), Solanaceae (Wollenweber et al, 1995). (3) Ginkgoaceae (Hou, et al, 2008), Eucryphiaceae (Wollenweber et al, 2000), Solanaceae (Wollenweber et al, 1995). 5,3'-diidroxi-7-4'-dimetoxiflavona (2) já foi isolada em Piper auritum (Ampofo, 1987) e Piper sylvaticum (Avijit \& Sudhir, 1982), além de outras famílias, tais como: Isoetaceae (Seijas et al, 2004), Thymelaeaceae (Wang et al, 2008), Eucryphiaceae (Wollenweber et al, 2000).

\section{MATERIAL E MÉTODOS}

\section{Métodos gerais}

Nas colunas cromatográficas foi utilizada como fase fixa Sephadex LH-20 e gel de sílica $(0,063-0,200 \mathrm{~mm}$, Merck) e nas placas cromatográficas em camada delgada analítica gel de sílica 60 PF 254 (Merck). Os compostos foram detectados usando luz UV ( $\lambda_{\max } 259$ e $\left.360 \mathrm{~nm}\right)$. Os espectros de IV foram obtidos em pastilha de $\mathrm{KBr}$ e os de $\mathrm{UV}$ em $\mathrm{MeOH}$ e $\mathrm{MeOH} / \mathrm{AlCl}_{3}$. Os espectros de $\mathrm{RMN}$ foram registrados a $200 \mathrm{MHZ}\left({ }^{1} \mathrm{H}\right)$ e $50 \mathrm{MHz}\left({ }^{13} \mathrm{C}\right)$ utilizando técnicas uni e bidimensionais. Os deslocamentos químicos foram relatados em ppm relativos a acetona- $\mathrm{d}_{6}$ a $27^{\circ} \mathrm{C}$. 


\section{Material botânico}

As partes aéreas da planta foram coletadas no Museu Emílio Goeldi, Belém-PA, onde uma exsicata da espécie está depositada sob o código MSP-009.

\section{Extração e isolamento}

O pó das partes aéreas de Piper carniconnectivum $(1300 \mathrm{~g})$ foi exaustivamente extraído com EtOH 95\% (4x 2 L). O extrato foi concentrado sob pressão reduzida e particionado com hexano, $\mathrm{CHCl}_{3}$ and AcOEt. A fase hexânica, após concentração em rotaevaporador, foi submetida a cromatografia em coluna de gel de sílica utilizando-se como eluente hexano, $\mathrm{CHCl}_{3}$, ACOEt e $\mathrm{MeOH}$ puros ou em misturas binárias, fornecendo 101 frações. As frações 55-59 foram novamente submetidas a cromatografia em coluna com gel de sílica $(0,063-0,200$ $\mathrm{mm}$, Merck) produzindo dezoito frações, dentre as quais as frações 6-7, que após purificação e análise dos espectros de $\mathrm{RMN}$ de ${ }^{1} \mathrm{H}$ e ${ }^{13} \mathrm{C}$, mostrou tratar-se da mistura dos esteróides $\beta$-sitosterol (A) e estigmasterol (B). A fase clorofórmica concentrada foi submetida a cromatografia em coluna de Sephadex LH-20 e MeOH. A fração 8 foi purificada em coluna de Sephadex e eluída também com metanol produzindo nove frações. As frações 3-6 e 9, submetidas a recristalização com uma mistura de $\mathrm{CHCl}_{3} \mathrm{e}$ $\mathrm{MeOH}$ (1:1), forneceu os compostos $\mathbf{1} \mathrm{e}$, respectivamente. As frações 15-17 da coluna inicial foram novamente submetidas a cromatografia em coluna de Sephadex e eluídas com $\mathrm{MeOH}$ resultando em dez frações. A fração 9, após eluição com MeOH em coluna de Sephadex LH-20, forneceu o composto 3 .

\section{RESULTADOS E DISCUSSÃO}

Os dados espectrais de RMN de ${ }^{1} \mathrm{H}$ e ${ }^{13} \mathrm{C}$ aliados a comparação com dados da literatura (Kojima et al, 1990; Tomaz et al, 2008) permitiram identificar a mistura dos esteróides $\beta$-sitosterol e estigmasterol (A e B).

$\mathrm{O}$ espectro de $\mathrm{RMN}$ de ${ }^{13} \mathrm{C}$, experimento APT, das substâncias 1 e $\mathbf{2}$ mostrou sinais para quinze carbonos, dentre os quais seis para carbonos metínicos ( $\delta$ 120,08,
$116,62,114,15,104,18,98,62,93,15$ para 1 ; e $\delta 121,35$, $116,34,110,45,104,41,98,62,93,20$ para 2 ), nove para carbonos quaternários $(\delta 183,12,166,50,165,37,162,97$, $158,60,150,00,146,57,123,49,106,00$ para $\mathbf{1}$; e $\delta 183,17$, $166,49,165,18,162,98,158,62,151,51,148,84,123,35$, 105,91 para 2), além de dois sinais para carbonos metílicos em $\delta 56,51$ e $\delta 56,34$ para 2 e um sinal em $\delta 56,35$ para 1 . O espectro de RMN de ${ }^{1} \mathrm{H}$ mostrou um singleto característico de $\mathrm{OH}-5$ em ponte de hidrogênio com carbonila da posição 4 de flavonas ( $\delta$ 12,98 para $\mathbf{1}$ e $\mathbf{2}$ ), sugestão reforçada pela presença de carbono metínico em $\delta 104,18$ e $\delta 104,41$ para os carbonos da posição 3 de 1 e 2 , respectivamente (Agrawal, 1989), além de dois dubletos característicos da presença de um par de hidrogênios acoplados em meta, para cada composto $[\delta 6,67(J=2,3 \mathrm{~Hz}, 1 \mathrm{H})$ e $6,30(J=$ $2,3 \mathrm{~Hz}, 1 \mathrm{H})$ para $\mathbf{1} ; \delta 6,70(J=2,2 \mathrm{~Hz}, 1 \mathrm{H})$ e $6,31(J=2,2$ $\mathrm{Hz}, 1 \mathrm{H})$ para 2]. Tais dubletos, juntamente com os seus carbonos correspondentes $(\delta 93,15$ e 98,62 para $1 ; \delta 93,20$ e 98,62 para 2 ), deduzidos com a ajuda do experimento HMQC, foram atribuídos às posições 6 e 8 do anel A dos dois compostos, visto que os valores dos deslocamentos químicos dos carbonos são característicos de carbonos vizinhos a dois outros com substituintes oxigenados (Asim, 2002). Essas atribuições foram corroboradas pela presença de correlação, no espectro HMBC, entre o singleto atribuído a $\mathrm{OH}-5$ e os valores de deslocamento químico atribuídos a C-6, C-10 e C-5 ( $\delta 12,98 / 98,62,106,00,162,97$ para 1, $\delta 12,98 / 98,62,105,91,162,98$ para 2). O experimento HMBC ainda mostrou correlação entre H-6 e os carbonos das posições 7,10 e $5(\delta 6,30 / 166,50,106,00,162,97$ para $1 ; \delta 6,31 / 166,49,105,91,162,98$ para 2 ) e entre H-8 e os carbonos das posições $7,9,10$ e $6(\delta 6,67 / 166,50,158,60$, 106,00 e 98,62 para $1 ; \delta 6,70 / 166,49,158,62,105,91$ e 98,62 para 2), o que confirmou os deslocamentos químicos para os carbonos $6,10,7$ e 5 e permitiu atribuir C-9. O mesmo espectro também mostrou as correlações entre H-3 e C-2 $(\delta 6,61 / 165,37 ; \delta 6,74 / 165,18)$, respectivamente para 1 e 2. Esses dados são sugestivos de que os dois compostos têm anéis $\mathrm{A}$ e $\mathrm{C}$ iguais.

$\mathrm{O}$ espectro de $\mathrm{RMN}$ de ${ }^{1} \mathrm{H}$ de $\mathbf{1}$ e $\mathbf{2}$ ainda revela sinais em $\delta 7,50(\mathrm{~d}, J=2,2 \mathrm{~Hz}, 1 \mathrm{H}), \delta 7,48(\mathrm{dd}, J=2,2$ e 8,0 $\mathrm{Hz}, 1 \mathrm{H}), 6,99(\mathrm{~d}, J=8,0 \mathrm{~Hz}, 1 \mathrm{H})$, para 1 e $\delta 7,64(\mathrm{sl}, 1 \mathrm{H})$, $\delta 7,62(\mathrm{dd}, J=2,0$ e $8,8 \mathrm{~Hz}, 1 \mathrm{H})$ e $\delta 7.0(\mathrm{~d}, J=8,8 \mathrm{~Hz}, 1 \mathrm{H})$

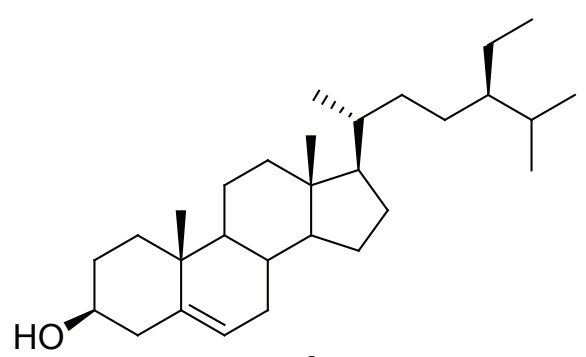

A<smiles>CC[C@H](/C=C/[C@H](C)[C@H]1CCC2C3CC=C4C[C@@H](O)CC[C@]4(C)C3CC[C@]21C)C(C)C</smiles>

B 
para 2, o que sugere anel aromático 1,3,4-trisubstituído, cujos sinais foram atribuídos para as posições 2', 6' e 5', respectivamente. Tal fato, juntamente com a presença de mais um sinal característico para metoxila aromática em $\delta 3,98(\mathrm{~s}, 3 \mathrm{H})$ para 2 permite sugerir a existência de hidroxila e metoxila como substituintes do anel B de 2 e duas hidroxilas no mesmo anel de $\mathbf{1}$. O espectro de HMQC permitiu atribuir os sinais $\delta 114,15,116,62$, 120,08 e $\delta 110,45,116,34,121,35$, com seus respectivos hidrogênios $(\delta 7,50 / 114,15, \delta 6,99 / 116,62, \delta 7,48 / 120,08 \mathrm{e}$ $\delta 7,64 / 110,45,7,00 / 116,34,7,62 / 121,35)$, para as posições 2', 5' e 6' de 1 e 2, respectivamente. Os experimentos HMBC registrados para $\mathbf{1}$ e $\mathbf{2}$ mostraram as correlações: $\delta 7.50\left(\mathrm{H}^{2}{ }^{\prime}\right) / 150,00, \delta 7,48\left(\mathrm{H}^{\prime} 6{ }^{\prime}\right) / 165,37$ e 146,57 e $\delta$ $6,99\left(\mathrm{H}-5^{\prime}\right) / 146,57,123,49$ para 1, além das seguintes: $\delta$ $7,64\left(\mathrm{H}-2^{\prime}\right) / 165,18$ e $151,51, \delta 7,62\left(\mathrm{H}^{-6}{ }^{\prime}\right) / 165,18,148,84$, $\delta 7.00\left(\mathrm{H}^{-5}{ }^{\prime}\right) / 148.84$ e 123,35 para 2 , o que permitiu atribuir $\delta 150,00,165,37,146,57$ e 123,49, além de $\delta$ $151,51,165,18,148,84$ e 123,35 para as posições 3', 2, 4' e 1', respectivamente de $\mathbf{1}$ e $\mathbf{2}$. O espectro 2D-NOESY de 2 mostrou correlação entre o sinal da metoxila em $\delta 3,91$ $(\mathrm{s}, 3 \mathrm{H}) \operatorname{com} \delta 6,31(\mathrm{H}-6)$ e $\delta 6,70(\mathrm{H}-8)$, o que permitiu deduzir que a absorção em $\delta 3,98$ corresponde a metoxila do anel B, o que foi evidenciado através da correlação, no mesmo espectro, entre $\delta 3,98 / \delta 7,62$.

$\mathrm{O}$ espectro de $\mathrm{RMN}$ de ${ }^{1} \mathrm{H}$ de 3 mostrou, para o anel $\mathrm{B}$, sinais típicos de um sistema AA'BB': $\delta 7,96$ $(\mathrm{dd}, J=8,8$ e $2,0 \mathrm{~Hz}, 2 \mathrm{H}), \delta 7,02(\mathrm{dd}, J=8,8$ e $2,0 \mathrm{~Hz}$, $2 \mathrm{H}$ ), os quais, juntamente com os sinais dos carbonos correspondentes, vistos no experimento HMQC, ou seja: $\delta 129,29$ e 116,85, respectivamente, foram atribuídos, com o auxílio da correlação $\delta 7,96 / 165,28$ (C-2) para as posições 2', 6' e 3', 5', respectivamente. Os sinais para carbonos não hidrogenados em $\delta 162,00$ e 183,17 foram atribuídos, respectivamente, para 4' e 4. Comparação dos dados de RMN de ${ }^{1} \mathrm{H}$ e ${ }^{13} \mathrm{C}$ de $\mathbf{3}$ com aqueles obtidos para 1 e 2 mostrou que os anéis A e C são idênticos para as três substâncias, diferindo apenas quanto ao padrão de substituição do anel B.<smiles>COc1cc(O)c2c(=O)cc(-c3ccc(O)c(O)c3)oc2c1</smiles><smiles>COc1cc(O)c2c(=O)cc(-c3ccc(OC)c(O)c3)oc2c1</smiles><smiles>COc1cc(O)c2c(=O)cc(-c3ccc(O)cc3)oc2c1</smiles>

(1) 5, 3',4'-triidroxi-7-metoxiflavona, pó amarelo (30 mg), p.f.: $266-268{ }^{\circ} \mathrm{C} ; v_{\max }\left(\mathrm{KBr}, \mathrm{cm}^{-1}\right)$ : 3417, 2924, 1653, 1597, 1498; UV ( $\left.\lambda_{\max }, \mathrm{nm}\right) \mathrm{MeOH}: 255,346 \mathrm{sh}$; UV ( $\left.\lambda_{\max }, \mathrm{nm}\right) \mathrm{MeOH}+\mathrm{AlCl}_{3}: 273$ sh. Dados $\mathrm{RMN}$ de ${ }^{1} \mathrm{H}\left(200 \mathrm{MHz}\right.$, acetona- $\left.\mathrm{d}_{6}\right)$ e ${ }^{13} \mathrm{C}$ (Tabela 1)

(2) 5, 3'-diidroxi-7, 4'-dimetoxiflavona, cristais em agulha amarelos $(15 \mathrm{mg})$, p.f.: $234{ }^{\circ} \mathrm{C} ; \mathrm{v}_{\max }(\mathrm{KBr}$, $\left.\mathrm{cm}^{-1}\right): 3.433,2.924,2.848,1.653,1.589,1.500$; UV ( $\left.\lambda_{\max }, \mathrm{nm}\right) \mathrm{MeOH}: 268,346 \mathrm{sh}$; UV $\left(\lambda_{\max }, \mathrm{nm}\right)$ $\mathrm{MeOH}+\mathrm{AlCl}_{3}: 275,360$ sh. Dados RMN de ${ }^{1} \mathrm{H}\left(200 \mathrm{MHz}\right.$, acetona-d $\left.\mathrm{d}_{6}\right){ }^{13} \mathrm{C}$ (Tabela 1).

(3) 5,4'-diidroxi-7-metoxiflavona , pó amarelo $(7 \mathrm{mg})$, p.f.: $286-288{ }^{\circ} \mathrm{C} ; v_{\max }\left(\mathrm{KBr}, \mathrm{cm}^{-1}\right)$ : 3.414, 2.924, 2.850, 1.664, 1.602, 1.500; UV ( $\left.\lambda_{\max }, \mathrm{nm}\right) \mathrm{MeOH}: 267,339 \mathrm{sh}$; UV ( $\left.\lambda_{\max }, \mathrm{nm}\right) \mathrm{MeOH}+\mathrm{AlCl}_{3}: 277,384$ sh. Dados RMN de ${ }^{1} \mathrm{H}\left(200 \mathrm{MHz}\right.$, acetona-d $\left.\mathrm{d}_{6}\right)$ e ${ }^{13} \mathrm{C}$ (Tabela 1$)$.

\section{AGRADECIMENTOS}

Os autores agradecem ao CNPq pelo suporte financeiro, a Dra. Elsie Guimarães pela identificação do material botânico e a Vicente Carlos Oliveira Costa pelos espectros de RMN. 
Tabela 1. dados de $\mathrm{RMN}$ de ${ }^{1} \mathrm{H}(200 \mathrm{MHz})$ e ${ }^{13} \mathrm{C}(50 \mathrm{MHz})$ e correlações obtidas em experimentos HMQC e HMBC registrados em acetona-d $_{6}, \mathrm{~J}(\mathrm{~Hz})$ e $\delta(\mathrm{ppm})$ para $\mathbf{1}, \mathbf{2}$ e $\mathbf{3}$.

\begin{tabular}{|c|c|c|c|c|c|c|c|c|c|}
\hline \multicolumn{7}{|c|}{ HMQC } & \multirow{2}{*}{\multicolumn{3}{|c|}{$\begin{array}{l}\text { HMBC } \\
{ }^{2,3} \mathrm{~J}(\mathrm{CH}) \\
\end{array}$}} \\
\hline & \multicolumn{3}{|c|}{$\delta \mathrm{C}$} & \multicolumn{3}{|c|}{$\delta \mathrm{C}$} & & & \\
\hline & 1 & 2 & 3 & 1 & 2 & 3 & 1 & 2 & 3 \\
\hline 1 & & & & & & & & & \\
\hline 2 & 165.37 & 165.18 & 165.28 & & & & H-3,H-6, & H-6',H-2', H-3 & H-2',H-6', H-3 \\
\hline 3 & 104.18 & 104.41 & 104.00 & $6.61(\mathrm{~s}, 1 \mathrm{H})$ & $6.74(\mathrm{~s}, 1 \mathrm{H})$ & $6.67(\mathrm{~s}, 1 \mathrm{H})$ & & & \\
\hline 4 & 183.12 & 183.17 & 183.17 & & & & & $\mathrm{H}-3$ & $\mathrm{H}-3$ \\
\hline 5 & 162.97 & 162.98 & 163.03 & & & & OH-5,H-6 & OH-5,H-6 & H-6 \\
\hline 6 & 98.62 & 98.62 & 98.63 & $\begin{array}{c}6.30(\mathrm{~d}, 1 \mathrm{H} \\
J=2,3 \mathrm{~Hz})\end{array}$ & $\begin{array}{c}6.31(\mathrm{~d}, 1 \mathrm{H} \\
J=2,2 \mathrm{~Hz})\end{array}$ & $\begin{array}{c}6.31(\mathrm{~d}, 1 \mathrm{H}, \\
J=2,2 \mathrm{~Hz})\end{array}$ & $\mathrm{OH}-5, \mathrm{H}-8$ & H-8, OH-5 & H-8 \\
\hline 7 & 166.50 & 166.49 & 166.53 & & & & H-6, OMe-7 & $\begin{array}{l}\text { OMe-7, H-6 } \\
\text { e } 8\end{array}$ & OMe-7, H-6 e 8 \\
\hline 8 & 93.15 & 93.20 & 93.17 & $\begin{array}{c}6.67(\mathrm{~d}, 1 \mathrm{H} \\
J=2,3 \mathrm{~Hz})\end{array}$ & $\begin{array}{c}6.70(\mathrm{~d}, 1 \mathrm{H} \\
J=2,2 \mathrm{~Hz})\end{array}$ & $\begin{array}{c}6.69(\mathrm{~d}, 1 \mathrm{H} \\
J=2,2 \mathrm{~Hz})\end{array}$ & & H-6 & H-6 \\
\hline 9 & 158.60 & 158.62 & 158.65 & & & & $\mathrm{H}-8$ & H-8 & H-8 \\
\hline 10 & 106.00 & 105.91 & 105.93 & & & & H-3,OH-5 & $\begin{array}{l}\text { H-6, H-8, } \\
\text { OH-5, H-3 }\end{array}$ & H-6, H-8 \\
\hline 1 , & 123.49 & 123.35 & 123.00 & & & & H-2', H-5', & H-5', H-6' H-3 & H-2',H-6', H-3 \\
\hline 2 & 114.15 & 110.45 & 129.29 & $\begin{array}{l}7.50(\mathrm{~d}, 1 \mathrm{H} \\
J=2,2 \mathrm{~Hz})\end{array}$ & $7.64(\mathrm{sl}, 1 \mathrm{H})$ & $\begin{array}{c}7.96(\mathrm{dd}, \\
2 \mathrm{H}, J=8,8 \\
\text { e } 2,0 \mathrm{~Hz})\end{array}$ & H-6 & H-6 & \\
\hline 3 , & 150.00 & 151.51 & 116.85 & & & $\begin{array}{c}7.02(\mathrm{dd}, \\
2 \mathrm{H}, J=8,8 \\
\text { e } 2,0 \mathrm{~Hz})\end{array}$ & $\mathrm{H}-2$ & H-5', H-2' & \\
\hline $4^{\prime}$ & 146.57 & 148.84 & 162.00 & & & $9.29(\mathrm{~s}, 1 \mathrm{H})$ & H-5, H-6' & $\begin{array}{l}\text { H-6',H-5', } \\
\text { OMe-4' }\end{array}$ & $\begin{array}{l}\text { H-2',H-6', } \\
\text { H-5', H-3', }\end{array}$ \\
\hline 5, & 116.62 & 116.34 & 116.85 & $\begin{array}{c}6.99(\mathrm{~d}, 1 \mathrm{H} \\
J=8,0)\end{array}$ & $\begin{array}{l}7.00(\mathrm{~d}, 1 \mathrm{H} \\
J=8,8 \mathrm{~Hz})\end{array}$ & $\begin{array}{c}7.02(\mathrm{dd}, \\
2 \mathrm{H}, J=8,8 \\
\text { e } 2,0 \mathrm{~Hz})\end{array}$ & & & \\
\hline 6 ' & 120.08 & 121.35 & 129.29 & $\begin{array}{c}7.48(\mathrm{dd}, \\
1 \mathrm{H}, J=8,0 \\
\text { e } 2,2 \mathrm{~Hz})\end{array}$ & $\begin{array}{c}7.62(\mathrm{dd}, \\
1 \mathrm{H}, J=2,0 \mathrm{e} \\
8,8 \mathrm{~Hz})\end{array}$ & $\begin{array}{c}7.96(\mathrm{dd}, \\
2 \mathrm{H}, J=8,8 \\
\text { e } 2,0 \mathrm{~Hz})\end{array}$ & & & \\
\hline OMe-7 & 56.35 & 56.34 & 56.36 & $3.91(\mathrm{~s}, 3 \mathrm{H})$ & $3.91(\mathrm{~s}, 3 \mathrm{H})$ & $3.91(\mathrm{~s}, 3 \mathrm{H})$ & & & \\
\hline OMe-4' & & 56.51 & & & $3.98(\mathrm{~s}, 3 \mathrm{H})$ & & & & \\
\hline OH-5 & & & & $\begin{array}{c}12,98(\mathrm{~s} \\
1 \mathrm{H})\end{array}$ & $12,98(\mathrm{~s}, 1 \mathrm{H})$ & $\begin{array}{c}12,98(\mathrm{~s} \\
1 \mathrm{H})\end{array}$ & & & \\
\hline $\mathrm{OH}-4$ & & & & & & $9.29(\mathrm{~s}, 1 \mathrm{H})$ & & & \\
\hline
\end{tabular}

\section{REFERÊNCIAS}

Agrawal PK 1989. Carbon-13 NMR of Flavonoids: Studies in Organic Chemistry 39. Lucknov, India: Elsevier.

Ahmad VU, Ali Z, Zahid M, Alan N, Saba N, Khan T 2000. Phytochemical study of Salvia moorcroftiana. Fitoterapia 71: 84-85.

Ampofo SA, Roussis V, Wiemer DF 1987. New prenilated phenolics from Piper auritum. Phytochemistry 26: 23672370.

Araújo-Jr JX, Chaves MCO, da Cunha EVL, Gray AI 1997. Piperdardine, a piperidine alkaloid from Piper tuberculatum. Phytochemistry 44: 559-561.
Araújo-Jr JX, Chaves MCO, da Cunha EVL, Gray AI 1999. Cepharanone b from Piper tuberculatum. Biochem Syst Ecol 27: 325-327.

Avijit B, Sudhir P 1982. Constituents of Piper sylvaticum: structure of Sylvatesmin. J Nat Prod 45: 672-673.

Asim M 2002. Isolation and structural studies in the chemical constituents of Salvia moorcroftiana and Euprorbia decipiens. International Centre for Chemical Sciences. Pakistan.

Bohm,BA 1999. Major exudate flavonoids of Dubautia arborea (Asteraceae). Biochem Syst Ecol 27: 755-757.

Cardozo-Júnior EL, Chaves MCO 2003. Caldensin, a new natural N-methylaristolactam from Piper caldense. Pharm Biol 


$$
\text { 41: 216-218 }
$$

Chaves MCO, Santos BV 2002. Constituents from Piper marginatum fruits Fitoterapia 73: 547-548.

Chaves MCO, Júnior AGF, Santos BVO 2003a. Amides from Piper tuberculatum fruits. Fitoterapia 74: 181-183.

Chaves MCO, Santos BVO, Oliveira AH 2003b. 1-Cinnamoyl pirrolidide from Piper marginatum. Biochem Syst Ecol 31: 1213-1214

Chaves MCO, Santos BVO, Oliveira AH 2006. Aristolactams from Piper marginatum Jacq. (Piperaceae). Biochem Syst Ecol 34: 75-77.

Cunha EVL, Chaves MCO 2001. Two amides from Piper marginatum fruits. Fitoterapia 72: 197-198.

Facundo VA, Sá AL, Silva SAF, Morais SM, Matos CRR, BrazFilho R 2003. Three new natural cyclopentenedione derivatives from Piper carniconnectivum. J Braz Chem Soc 15: 140-145.

Facundo VA, Braz-Filho R 2004. C-methylated flavonoids from the roots of Piper carniconnectivum C.DC. (Piperaceae). Biochem Syst Ecol 32: 1215-1217.

Hou D, Song S, Song M, Zang Yi-X, Li X, Wang JH 2008. Verification of bilobalide's absolute configuration using CD spectrum and chemical constituents of the extract from Ginkgo biloba. Shenyang Yaoke Daxue Xuebao 25: 290-293, 320 apud Chemical Abstracts 149:5231.

Kojima H, Sato N, Hatano A, Ogura H 1990. Sterol glucosides from Prunella vulgaris. Phytochemistry 29: 2351-2355.

Parmar VS, Jain SC, Bisht KS, Jain R, Taneja P, Jha A, Tyagi OD, Prasad AK, Wengel J, Olsen CE, Boll PM 1997. Phytochemistry 46: 597-673

Santos BVO, Chaves MCO, da Cunha EVL, Gray AI 1997. Croweacin from Piper marginatum. Biochem Syst Ecol 25: 471-472.

Santos BVO, Chaves MCO, da Cunha EVL, Gray AI 1998a. Phenyl alkanoids from Pipermarginatum. Phytochemistry 49: 1381-1384.

Santos BVO, Chaves MCO 1998b. Assignments of ${ }^{1} \mathrm{H}$ and ${ }^{13} \mathrm{C}$ ressonance signals in 2-methoxy-4,5methilenedioxypropiophenone with the assistance of 1D and 2D NMR experiments Acta Farm Bonaerense 19: 45-47.

Santos BVO, Chaves MCO 1999a. (E,E)-N_isobutyl-2,4octadienamide from Piper marginatum. Biochem Syst Ecol 27: 113-114.

Santos BVO, Chaves MCO 1999b. 2,4,5-trimethoxypropiophenone from Piper marginatum. Biochem Syst Ecol 27: 539-541.

Seijas JA, Vazquez-Tato MP, Crecente-Campo J, Ramil-Rego P, Romero MI, Fernandez P 2004. Flavonoids from the roots of Isoetes longissimum. Int Elect Con Synthetic Org Chem 1-30: 935-937.

Tomaz ACA, Nogueira RBSS, Pinto DS, Agra MF, Souza MFV, da-Cunha EVL 2008. Chemical constiuents from Richardia grandiflora (Cham. \& Schltdl.) Steud. (Rubiaceae). Rev Bras Farmacogn 18: 47-52.

Wang HG, Zhou MH, Lu JJ, Yu BY 2008. Antitumor constituents from the leaves of Aquilaria sinensis (Lour.) Gilg. Linchan Huaxue Yu Gongye 28: 1-5.

Wollenweber E, Doerr M 1995. Exsudate flavonoids in some Solanaceae. Biochem Syst Ecol 23: 457-458.

Wollenweber E, Dorr M, Rozefelds AC, Minchin P, Forster PI 2000. Variation in flavonoid exudates in Eucryphia species from Australia and South America. Biochem Syst Ecol 28: 111-118.

Yuncker TG 1972. The Piperaceae of Brazil. Hoehnea 2: 19366. 